\title{
Performance of WSN by Minimizing Delay and Maximizing Lifetime
}

\author{
P.V. Deshpande \\ PG Student \\ M.B.E.S's College of \\ Engineering, Ambajogai \\ Maharashtra, India
}

\author{
B. M. Patil \\ Professor M.B.E.S's College of \\ Engineering, Ambajogai \\ Maharashtra, India
}

\author{
V. M. Chandode \\ Professor M.B.E.S's College of \\ Engineering, Ambajogai \\ Maharashtra, India
}

\begin{abstract}
Efficient use of energy in Wireless Sensor Network to extends the network lifetime. In order to improve the lifetime of the Wireless Sensor Network. Sleep-wake scheduling is a valuable mechanism to extend the lifetime of these energycontrolled Wireless Sensor Networks. Conversely, sleepwake scheduling might result in extra delays because a transmitting node needs to wait for its next-hop relay node to wake up. In Literature lot of people used Sleep-wake up Scheduling algorithm in order to improve the lifetime of Wireless Sensor Network. But it requires extra delays because a transmitting node needs to wait for its next hop relay node to wakeup. To reduce these delays hence In this Work We Combined Anycast forwarding scheme and Sleep Wake up scheduling. Experimental evaluation shows that this method is better than alone Sleep-wake Scheduling. The comparison shows that the delays are minimized. and also It has been observed that packet delivery ratio and network life-time increases as increase number of nodes.
\end{abstract}

\section{Keywords}

Wireless Sensor Network, Anycast, Sleep-wake scheduling, Energy, Delay

\section{INTRODUCTION}

Wireless Sensor Network (WSN) is a network consisting of large numbers of wireless sensor nodes which collect the information from their surrounding environment and send the data to sink node. Due to Inexpensive nature, limited size and weight, Redundant nature Sensor nodes are having the limited battery capacity for that we have to acquire a solutions to keep network active for the longest period of time. In such event driven sensor network there are four source of energy consumption. These are communication radios on, data transmission and reception, sensors are active, transmission and reception of control packet. There are also Wasteful Energy consumption such as Idle listing, Retransmitting due to collision, overhearing and overemitting. Generally, WSNs operate for a long time in idle mode and only occasionally send data. The energy consumption of listening to the idle channel is equivalent to energy consumption when sending or receiving, and much larger than the energy consumption of the sleep mode. To receive data, in active/listen state the receiver must be in high power state, as in sleep state, the radio is in low power mode switched off. By putting nodes to sleep when there are no events, whenever communication is not required. Ideally, the radio should be switched off as soon as there is no more data to send/receive and should be resumed as soon as a new data packet becomes ready. To overcome this problem, mainly there are three protocols that are discussed in the next chapter.[1]

\section{LITERATURE REVIEW}

Several different sleep-wake scheduling protocols have been proposed in the literature. Among this one is Synchronized sleep-wake scheduling protocol. In this protocol, sensor nodes exchange the synchronization information with neighboring nodes periodically. due to this method also incurs additional overhead and consumes large amount of energy. On-demand sleep-wake scheduling protocol is one scheduling where nodes turn off most of their circuitry and always turn on a secondary low-powered receiver to listen to "wake-up" calls from neighboring nodes. because of, this on-demand sleepwake scheduling can significantly increase the cost of sensor node due to the additional receiver.

This work is concentrated on asynchronous sleep wake-up scheduling protocols where each node wakes up independently of neighboring node in order to save the energy But due to this independence of wake-up processes, extra delays encounter at each node along path to sink node because each node has to wait for its next hop node to wake up before transmitting the packet.[2]

In conventional packet-forwarding schemes, every node has one designated next-hop relaying node in the neighborhood, and it has to wait for the next-hop node to wake up when forward a packet has to be done In contrast, under any-cast packet-forwarding schemes, each node has multiple next-hop relaying nodes in a candidate set and forwards the packet to the first node that wakes up in the forwarding set. It is easy to see that, compared to the basic scheme anycast clearly reduces the expected one-hop delay. However, anycast does not necessarily lead to the minimum expected end-to-end delay because a packet can still be relayed through a timeconsuming routing path.[3]

Schurgers, et Al.[4] have proposed to reduce the energy consumption significantly.a node should turn off its radio most of the time, except when it has to participate in data forwarding. Kim, and Shrof presented in [5] that most efficient method to save energy in wireless sensor networks (WSNs) is to put nodes to sleep when there is no need to relay or transmit packets. Such mechanisms are called sleep-wake scheduling. Each node sleeps for a specified amount of time, then wakes up and listens to see if any other nodes want to communicate. Sleep-wake scheduling can significantly increase the packet-delivery delay because, at each hop, an event-reporting packet has to wait for its next-hop node to wake up. 


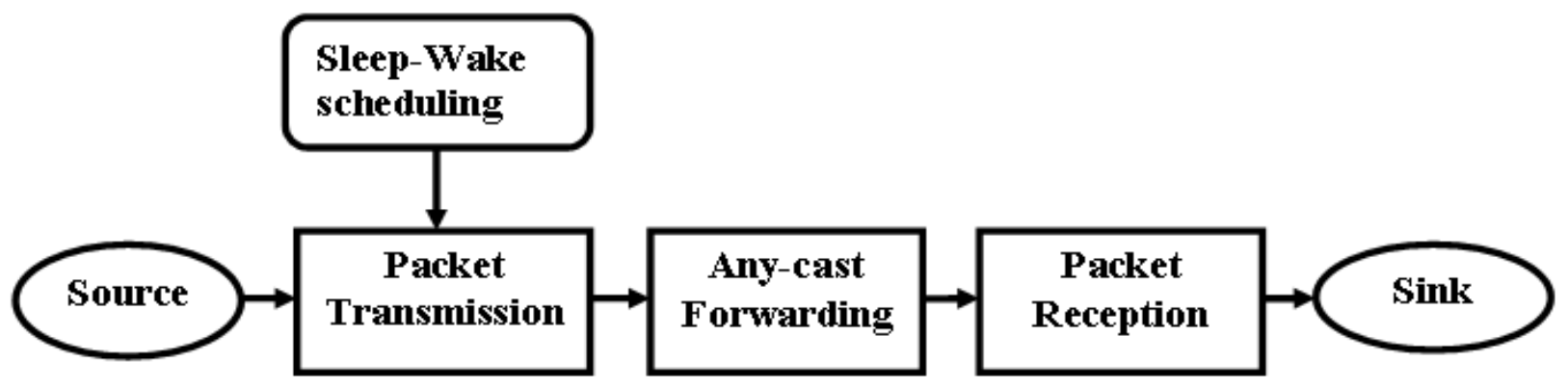

Fig 1: System Model

Polastre, and Culler[6]-[9] presented S-MAC low power RTSCTS protocol for wireless sensor networks inspired by PAMAS and 802.11. S-MAC periodically sleeps, wakes up, listens to the channel, and then returns to sleep. Each active period is of fixed size, $115 \mathrm{~ms}$, with a variable sleep period. The length of the sleep period dictates the duty cycle of SMAC. At the beginning of each active period, nodes exchange synchronization information.

Choudhury and Vaidya,[10] presented developing anycastbased packet forwarding schemes, where each node opportunistically forwards a packet to the first neighboring node that wakes up among multiple candidate nodes. The anycast forwarding schemes for minimizing the expected packet-delivery delays from the sensor nodes to the sink.

Kim [11] presents a solution to the combine control problem of how to optimally control the system parameters of the sleep-wake scheduling protocol and the anycast packetforwarding protocol to maximize the network lifetime, subject to a constraint on the expected end to end packet delivery delay.

\section{SYSTEM MODEL}

In scenario, This will discuss about system model, problem definition and algorithm. Fig1 dictates the system model regarding sleep wake scheduling. Let consider $\mathrm{N}$ nodes in a wireless sensor network. Each sensor node will detect events and relay packets. If a node detects an event it packs that event information into a packet and delivers to sink $\mathrm{S}$ via multi-hop relaying. Let assume that every node has at least one multi-hop path to the sink and there is a single sink. During packet transmission sleep wake scheduling is applied. in this sensor nodes sleep for most of the time and occasionally wake up for a short period of time t. The main goal of this Paper is, reducing the energy consumption during the transmission and reception, by minimizing number of active node in order to maximize network lifetime and reduces end to end delay.[12]

The fundamental solution to this problem is Anycast. packet forwarding should be implemented along with sleep scheduling..Here in order to get good performance. Two main challenges that have to be addressed in enhancing the performance of event driven wireless sensor network are:

\section{- Delay Minimization Problem}

With the wakeup rates of sensor nodes, optimally choosing the Anycast forwarding policy to reduce the expected delay.

\section{- $\quad$ Lifetime Maximization Problem}

With constraint on the expected end-to-end delay, finding ways to maximize The network lifetime by controlling both Wakeup rates and anycast packet forwarding policy.

\subsection{Sleep-wake Scheduling with Anycast}

Consider wireless sensor network in which every node broadcast the message to the neighboring nodes \& this process continues in operation when desired destination is encountered. Each sensor node follows a periodic sleep-wake up schedule and goes to sleep for some time and then wake up and listens to hear if any other node wants to talk to it. During sleep mode, the node turns off its radio, and sets a timer to wakeup itself later. The sleeping and listening time duration is selected before-hand based on application In this way nodes alternate between active and sleep periods depending on network activity. This behavior is usually referred to as duty cycling and duty cycle is defined as the fraction of time nodes are active during their lifetime [13]-[16].

As sensor nodes perform a cooperative task, they need to coordinate their sleep/wakeup times. This scheme requires synchronization among neighbor nodes. Nodes exchange their schedules by broadcasting it to all neighbor nodes. Use RTS (Request to send) and CTS (Clear to send), The node, which sends out the RTS packet, wins the medium, and the receive will reply with a CTS packet. When a node hears an RTS or CTS transmission from its neighbor, it will wake up shortly at the end of the transmission and if it is the next hop on the data own path, this will reduce the latency as it can immediately forward the packet without waiting the next scheduled awake period.[17]

Now we will discuss algorithm for sleep wakeup scheduling.

\section{Algorithm ()}

\{

Input: Wake Interval

Output: sleep/wakeup schedule

Begin

\{

Step 1: pkt _transmission_start()

Step 2: follow the determined sleep/wake schedule and set wake interval of the first hop neighbors of event

Step 3: $\quad$ send the message about the changed wake interval to the first hop neighbors.

Step 4: Change of sleep/wake interval by the first hop neighbor upon receipt of the updated sleep/wake schedule 
Step 5: wait of the event occurrence node for the arrival of next scheduled slot

Step 6: Send the data to the next hop neighbor using three way communication RTS, CTS and DATA.

\subsection{Maximization of Network Lifetime}

The network lifetime is defined as the time period in which all nodes of the network are communicating and alive (enough energy in their battery). Lifetime of the network is the sum of the energy consumption in the transmission, reception, sleep and idle mode of operation, and cannot exceed its initial energy.

Let $Q_{i}$ denote the energy available to node i. We assume that node $\mathrm{i}$ consumes $\mu_{i}$ units of energy each time it wakes up. $\lambda_{i}$ is the wake up rate of node $i$. So expected lifetime of node $i$ is defined as

$$
Q_{i} / \mu_{i} \lambda_{i}
$$

We define network lifetime as the shortest lifetime of all nodes. In other words, the network lifetime for a given awake probability vector is given by

$$
T(p)=\min _{i \in N} T_{i}(\vec{p})
$$

all nodes to be the same value $T$. Each node finds the probability $p_{i}$ that satisfies the target lifetime.[18]

\section{SIMULATION RESULT}

For experimental observations (NS-2) network simulation is used. NS is an object oriented discrete event simulator. The table 1.1 below shows the required parameter values during experimental evaluation. The results were analyzed by considering no of nodes 200, 250, 300,350,400. 450,500 We have considered initial energy for each node about 100 joules.

Table 1: Simulation Parameters

\begin{tabular}{|l|l|}
\hline $\begin{array}{l}\text { Simulation } \\
\text { Parameters }\end{array}$ & Values \\
\hline Number of nodes & $200,250,300,350,400,450,500$ \\
\hline $\begin{array}{l}\text { Transmission } \\
\text { power(Tx) }\end{array}$ & 0.3 \\
\hline $\begin{array}{l}\text { Receiving } \\
\text { power(Rx) }\end{array}$ & 0.6 \\
\hline Topology area & $670 \mathrm{~m} \times 670 \mathrm{~m}$ \\
\hline
\end{tabular}

\begin{tabular}{|l|l|}
\hline Initial energy & 100 joules \\
\hline Traffic type & CBR over UDP, TCP over FTP \\
\hline Channel type & Wireless channel \\
\hline Type of antenna & Omni-directional \\
\hline
\end{tabular}

Simulations show the comparative performance in both the networks. In the simulation environment, initially the packets are transmitted by node at constant bit rate .The size of packets are 1000bytes. The simulation is run for 5 seconds

\subsection{Result and Analysis}

The performance of Combined Anycast and Sleep wakeup Scheduling compared against the Sleep-wake up schedule. Experimental parameters, such as average delay per packet, energy per packet, Packet delivery ratio, are used to measure the performance of both.

\subsubsection{End to End Delay}

End to End Delay is referred to as the time span between the packet sent from a sensor node and packet received at the sink node. Delay values are measured by changing the number of sensor nodes from 200 to 500 .

End to End delay = $\underline{\text { Sacket arrival time -sent packet time }}$ total no of connection pairs

To minimize delay at each hop because node not to wait for long time for the wakeup interval of the next hop. As a result, average delay per packet in combined sleep-wake and anycast is less than sleep-wake schedule. As shown in Fig 2, the average delay experienced by the proposed system is the less than that of anycast.

\subsubsection{Average Energy}

Average energy per packet is a measure of energy spent for forwarding a packet to the sink node. It is an indicator of the lifetime that can be achieved by the protocols. In Figure 3, average energy per packet is plotted on y-axis, with varying number of sensor nodes (from 200 to 600) It can be observed that the average energy consumption per packet for the proposed system protocol is less than that of sleep-wake scheduling. .By doing so, in proposed system avoids the case where the nodes remain awake and stay idle as no traffic is to be forwarded. So the remaining average energy of proposed system is more than that of sleep-wake scheduling 


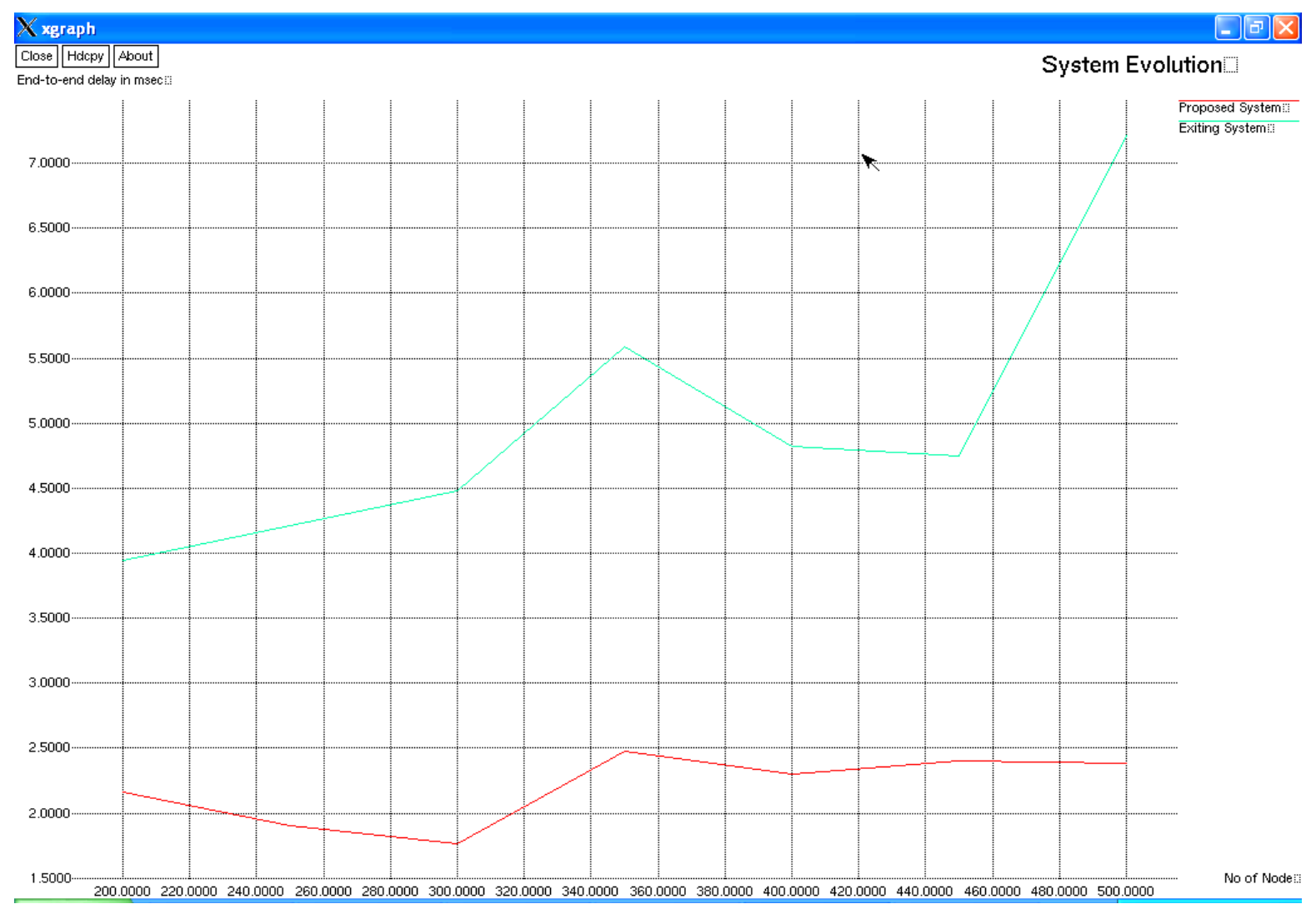

Fig 2: Average delay per packet for different number of sensor nodes

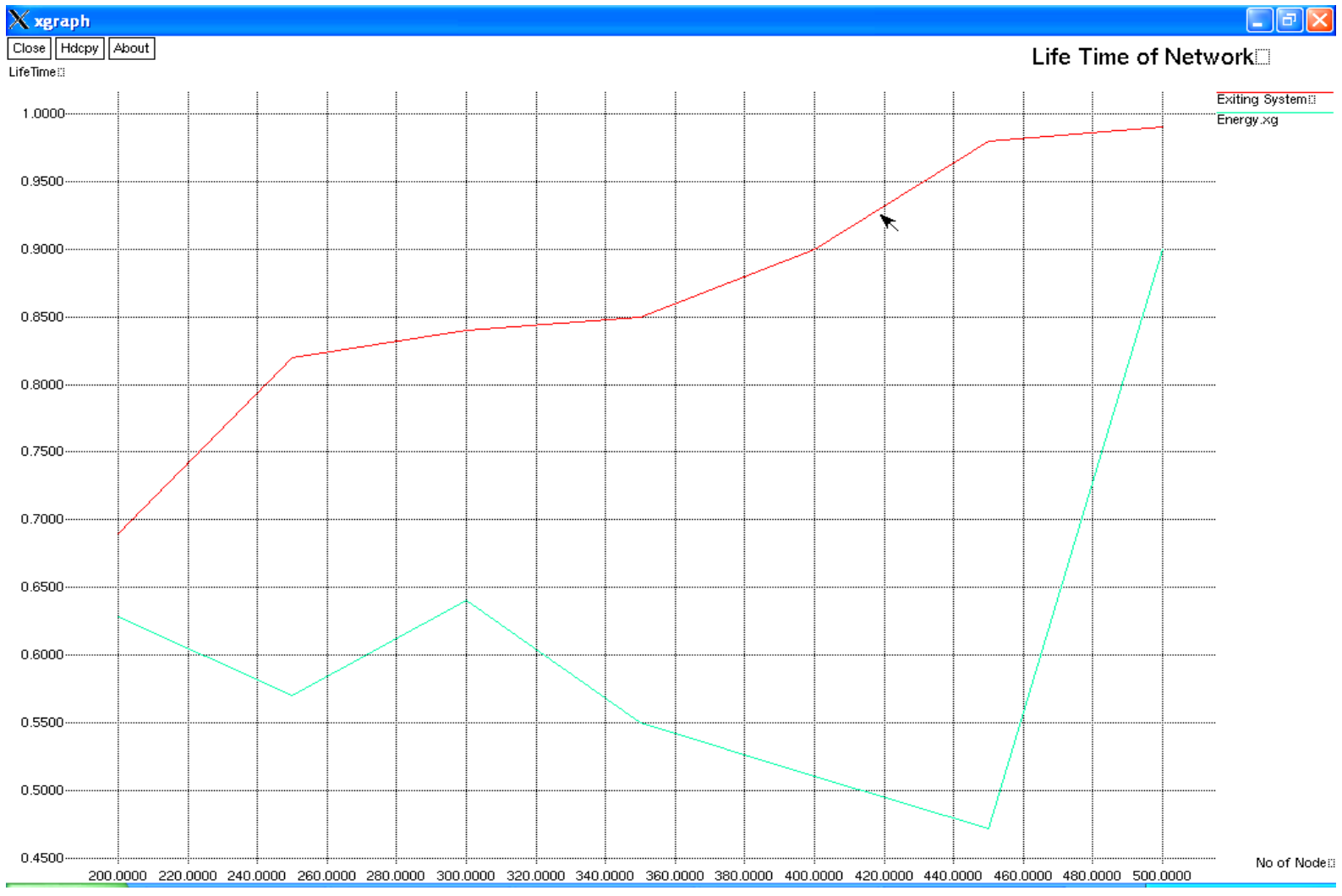

Fig 3 :Average Energy per packet for different number of sensor node 


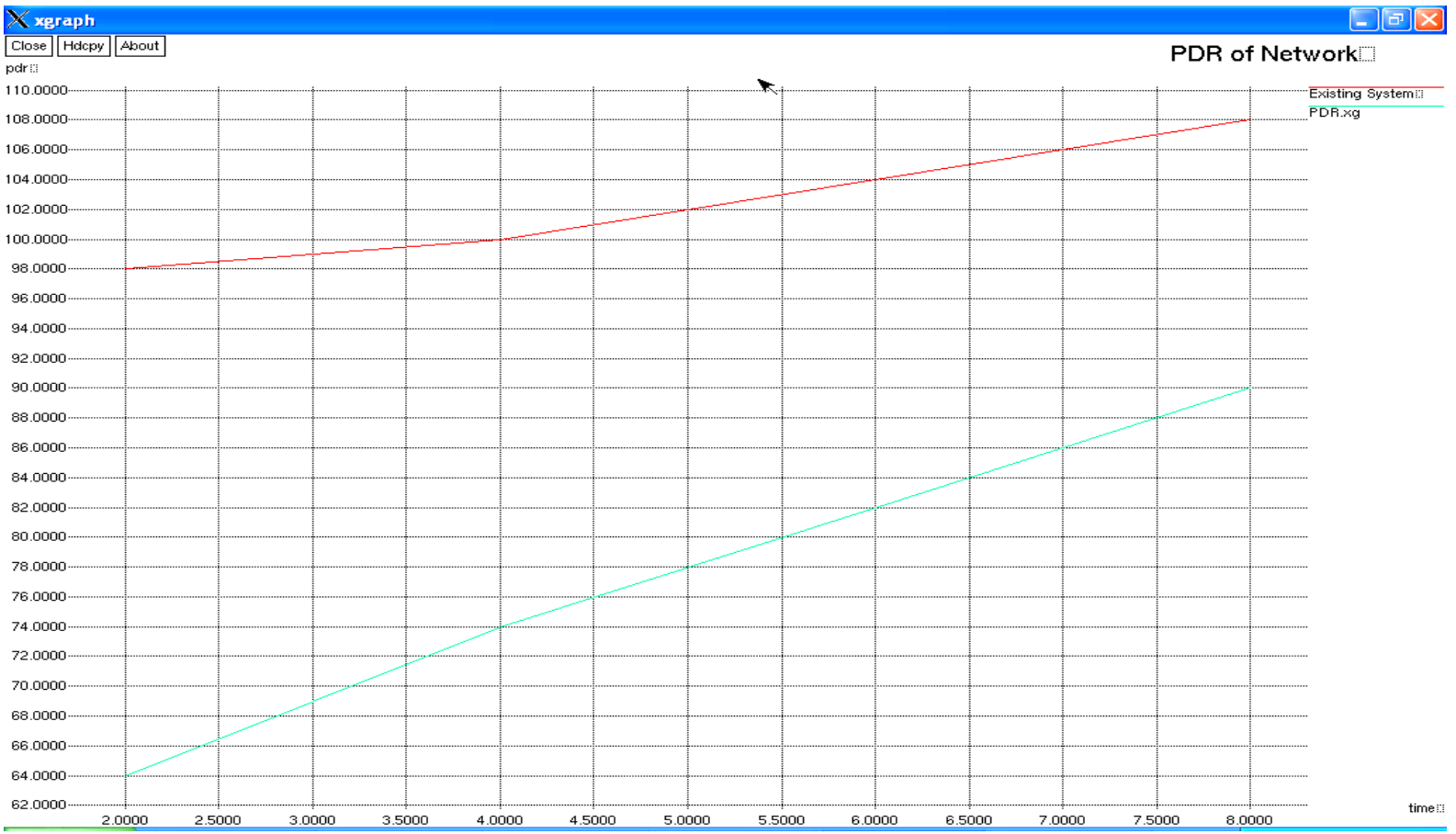

Fig 4: Packet Delivery Ratio per packet for different Time interval

\subsubsection{Packet Delivery Ratio}

Packet Delivery Ratio (PDR) is the ratio of total number of packets received successfully and the total number of

packets transmitted. As wake-up time increases packet delivery ratio increases

$p d r=\frac{\sum \text { No.of received data packets }}{\sum \text { No.of sent data packets }}$

Fig 4 shows comparison between Exiting System and Sleep wake scheduling along with anycast in terms of packet delivery ratio. The greater the value of packet delivery ratio means the better performance of the network.

\subsubsection{Lifetime of the Network}

The lifetime of the network is the sum of the energy consumption in the transmission, reception, sleep and idle mode of operation, and cannot exceed its initial energy, Hop count is the minimum number of hops to reach the sink of neighboring nodes to reduce the end-to-end delay. The objective of this algorithm is to minimize the time for a packet to advance one hop closer to the sink. Fig 5 shows comparison, Exiting System and Proposed System. As number of node increases the lifetime of network slightly increases in sleep-wake scheduling along with anycast.

\subsubsection{Performance of the network}

We observe that the Combined Sleep-wake up Scheduling and anycast algorithm can significantly reduce the delay compared with Sleep -wake Scheduling. Fig 6 shows comparison between network lifetimes subject to allowable delay. In Lifetime maximization Problem, we assume that the initial amount of energy is given by one-third of that in the end to end delay the same amount of energy each time when node wake up. From Table 2 value shows that comparison between existing system and proposed system. From that table we conclude that sleep-wake scheduling along with anycast minimize delay and maximize lifetime in WSN [16].

Table 2: Performance comparison between existing and proposed system

\begin{tabular}{|l|l|l|l|l|}
\hline \begin{tabular}{l} 
No $\begin{array}{l}\text { of } \\
\text { nodes }\end{array}$ \\
\cline { 2 - 5 }
\end{tabular} & \multicolumn{2}{|l|}{$\begin{array}{l}\text { Exiting } \\
\text { System }\end{array}$} & \multicolumn{2}{l|}{$\begin{array}{l}\text { Proposed } \\
\text { System }\end{array}$} \\
\cline { 2 - 5 } & Delay & Energy & Delay & Energy \\
\hline 200 & 3.94 & 0.6277 & 2.16 & 0.69 \\
\hline 250 & 4.211 & 0.57 & 1.9 & 0.82 \\
\hline 300 & 4.48 & 0.64 & 1.77 & 0.84 \\
\hline 350 & 5.59 & 0.55 & 2.48 & 0.85 \\
\hline 400 & 4.82 & 0.51 & 2.3 & 0.9 \\
\hline 450 & 4.75 & 0.47 & 2.4 & 0.98 \\
\hline 500 & 7.21 & 0.9 & 2.38 & 0.99 \\
\hline
\end{tabular}




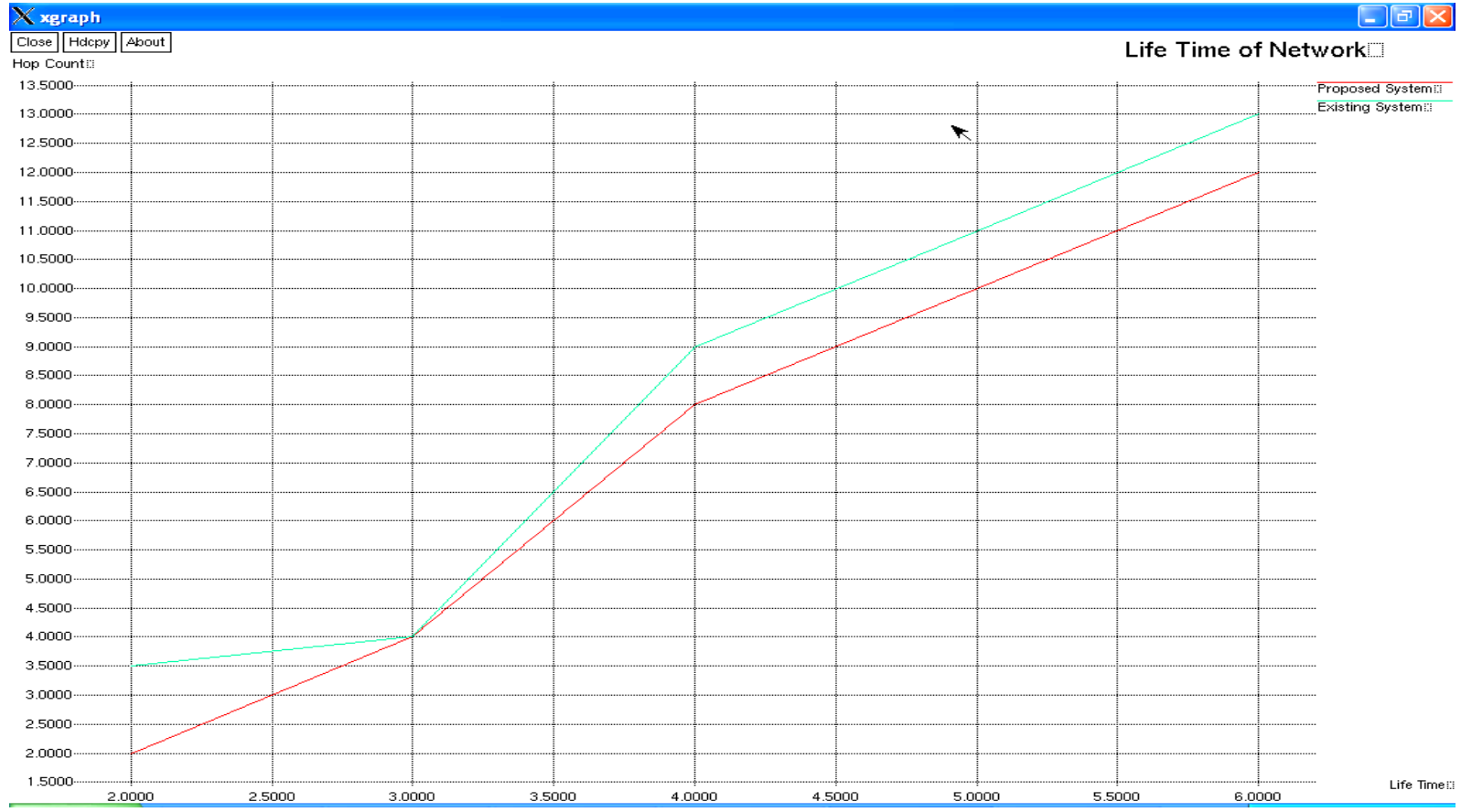

Fig 5: Hop count v/s Network lifetime

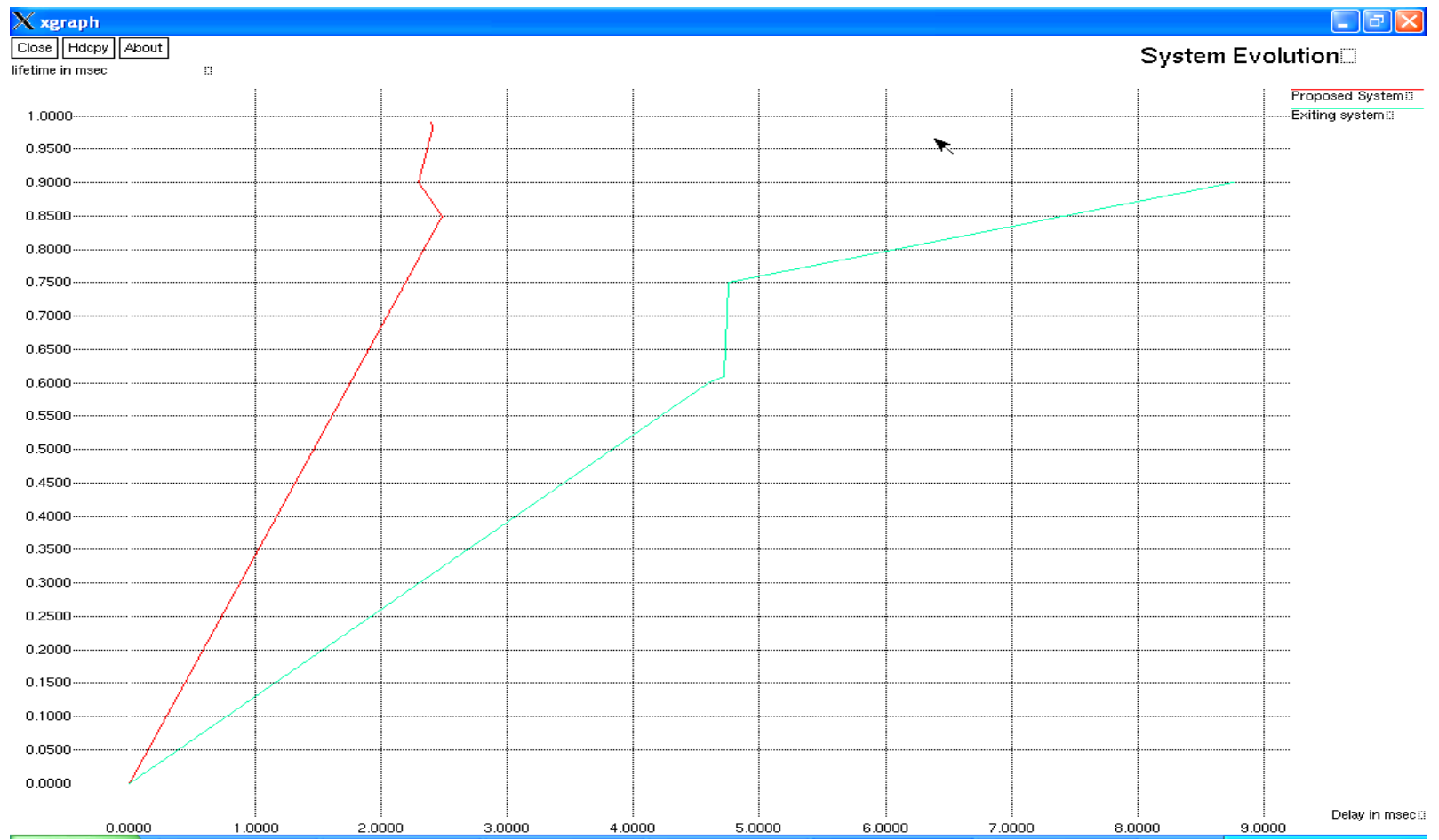

Fig 6: The network lifetime subject to different allowable delay

\section{CONCLUSION AND FUTURE SCOPE}

In wireless sensor networks Efficient utilization of the energy is very important. where nodes operate on limited battery. One of the main characteristics of these networks is that the network lifetime In the event driven wireless sensor network, Anycast along with sleep wakeup scheduling is designed to reduce the event-reporting delay and to extend the lifetime of wireless sensor networks by employing asynchronous sleepwake scheduling..Simulations are carried out to evaluate the performance of Sleep wake scheduling by comparing its performance with optimal anycast. Simulation results demonstrated that Optimal Anycast significantly reduced the end to-end delay, as well as improved the other parameters of average energy per packet, average delay, packet delivery ratio and maximize Network lifetime.

Future plan is to extend the simulations to consider other parameters and scenarios, such as throughput, fault tolerance, impact of aggregation, etc. Other important future extension is to evaluate the performance of Anycast along with sleep-wake up scheduling. 


\section{REFERENCES}

[1] J. Kim, X. Lin, N. Shroff and P. Sinha, "On maximizing the lifetime of delay-sensitive wireless sensor networks with anycast," in Proceedings of IEEE INFOCOM, pp. 807-815, Apr.2008.

[2] J.Chang and L.Tassiulas, "Maximum Lifetime Routing in Wireless Sensor Networks," in IEEE/ACM Transactions, vol.2 issue no.4, Aug. 2004.

[3] B.Nazir1,H.Hasbulla and S. Madani,"Sleep/wake scheduling scheme for minimizing end-to-end delay in multi-hop wireless sensor networks," Nazir et al. EURASIP Journal on Wireless Communications and Networking 2011.

[4] C. Schurgers, V. Tsiatsis, S. Ganeriwal, and M. Srivastava," "Optimizing sensor networks in the energy latency- density design space," IEEE Transaction on MOBILE COMPUTING, vol.1, issue no.1, pp.70-80, Mar.2002.

[5] J.Kim, X..Lin, and N.Shroff," "Optimal Anycast Technique for Delay-Sensitive Energy-Constrained Asynchronous Sensor Networks," IEEE INFOCOM, 2009

[6] Y.Tseng, C. Hsu, and T. Hsieh, "Power-saving protocols for IEEE 802.11-based multi-hop ad hoc networks," Computer Networks, vol.43, pp. 317337, Oct.2003.

[7] R. Choudhury and N.Vaidya, "MAC-layer anycasting in ad hoc networks," in SIGCOMM Computer Commununication., vol.34, Jan.2004.

[8] E. Shih, S.Cho, N. Ickes, R. Min, A. Sinha, A. Wang, and A. Chandrakasan, "Physical layer driven protocol and algorithm design for energy-efficient wireless sensor networks," in Proceedings of MOBICOM, pp.272-287, 2001.

[9] J. Polastre, J. Hill and D. Culler, "Versatile low power media access for wireless sensor networks," in Proceedings of SENSYS, pp.95-107, Nov.2004.

[10] J .Polastre, J Hui, P. Levis, J. Zhao, D. Culler, S. Shenker, and I. Stoica, "A unifying link abstraction for Wireless sensor networks," in Proceedings of SENSYS , pp.76-89, Nov.2005.

[11] J. Kim, X. Lin, N. Shroff and P. Sinha, "Minimizing Delay and Maximizing Lifetime for Wireless Sensor Networks with Anycast," in IEEE/ACM Transactions on NETWORKING, vol.18, issue no.2, Apr.2010.

[12] S. Liu, K. Fan and P. Sinha, "CMAC: An energy efficient MAC layer protocol using convergent packet forwarding for wireless sensor networks," in Proceedings of SECON, pp.11-20, Jun.2007.

[13] P. Larsson and N. Johansson, "Multiuser diversity forwarding in multi-hop packet radio networks," in Proceedings of IEEE WCNC, vol.4, pp.2188-2194, 2005.

[14] S. Biswas and R. Morris, "ExOR: Opportunistic multi-hop routing for wireless networks," in Proceedings of ACM SIGCOMM, vol.35, pp.133144, Oct. 2005.

[15] M. Rossi and M. Zorzi, "Integrated cost-based MAC and routing techniques for hop count forwarding in wireless sensor networks," in IEEE Transaction on MOBILE COMPUTER, vol.6, no. 4, pp.434-448, Apr.2007.

[16] J.Chang and L. Tassiulas, "Energy conserving routing in wireless ad-hoc networks," in Proceeding. IEEE INFOCOM, vol.1, pp.22-31, Mar. 2000.

[17] M.Elaneizi, "Prolonging Network Lifetime of clustered wireless sensor network," A thesis presented to the University of Waterloo in fulfillment of the thesis requirement for the degree of Mater of Applied Science in Electrical and Computer Engineering Waterloo, Ontario, Canada, 2008

[18] C.Kartal, "Energy efficiency and routing in sensor networks," Institute for Elektroniske Systemer, Aalborg University Publication date: 2011. 